\title{
SOME NEW EXISTENCE, SENSITIVITY AND STABILITY RESULTS FOR THE NONLINEAR COMPLEMENTARITY PROBLEM*
}

\author{
RUBÉN LÓPEZ ${ }^{1}$
}

\begin{abstract}
In this work we study the nonlinear complementarity problem on the nonnegative orthant. This is done by approximating its equivalent variational-inequality-formulation by a sequence of variational inequalities with nested compact domains. This approach yields simultaneously existence, sensitivity, and stability results. By introducing new classes of functions and a suitable metric for performing the approximation, we provide bounds for the asymptotic set of the solution set and coercive existence results, which extend and generalize most of the existing ones from the literature. Such results are given in terms of some sets called coercive existence sets, which we also employ for obtaining new sensitivity and stability results. Topological properties of the solution-set-mapping and bounds for it are also established. Finally, we deal with the piecewise affine case.
\end{abstract}

Mathematics Subject Classification. 90C31, 90C33, 47J20, 49J40, 49J45.

Received July 12, 2006. Revised January 15, 2007.

Published online January 18, 2008.

\section{INTRODUCTION}

Let $F: \mathbb{R}_{+}^{n} \rightarrow \mathbb{R}^{n}$ be a given function, and let $\mathbb{R}_{+}^{n}$ be the set of vectors from $\mathbb{R}^{n}$ with nonnegative components. The nonlinear complementarity problem in $\mathbb{R}^{n}$, denoted by $\operatorname{NCP}(F)$, is the following:

$$
\text { find } \bar{x} \in \mathbb{R}_{+}^{n} \text { such that } F(\bar{x}) \in \mathbb{R}_{+}^{n} \text { and }\langle F(\bar{x}), \bar{x}\rangle=0 .
$$

If we set $F(x)=M x+q$ where $M \in \mathbb{R}^{n \times n}$ and $q \in \mathbb{R}^{n}$, then the problem is called the linear complementarity problem (LCP). These problems are important in various equilibrium settings and has wide range of applications in Science and Engineering (see $[3,8,15,19,22,32]$ and the references therein).

Problem (NCP) is known to be equivalent to the following variational inequality problem $\operatorname{VIP}\left(\mathbb{R}_{+}^{n}, F\right.$ ) (see Lem. 3.1 from [23]), which will serve as our main framework for its study.

$$
\text { find } \bar{x} \in \mathbb{R}_{+}^{n} \text { such that }\langle F(\bar{x}), x-\bar{x}\rangle \geq 0 \quad \forall x \in \mathbb{R}_{+}^{n} .
$$

Sensitivity and stability analysis of the $\operatorname{NCP}(F)$ is concerned with the study of the behavior of the solution(s) of this problem when the data are subject to change. This analysis provide valuable qualitative information

\footnotetext{
Keywords and phrases. Nonlinear complementarity problem, variational inequality, asymptotic analysis, sensitivity analysis.

* This work has been supported by CONICYT-Chile through Proyecto FONDECYT de Iniciación en Investigación 11060015 and Proyecto DIN-03/2006 Universidad Católica de la Santísima Concepción.

${ }^{1}$ Facultad de Ingeniería, Universidad Católica de la Santísima Concepción, Concepción, Chile; rlopez@ucsc.cl
} 
on the problem. In the literature we can find different approaches for obtaining sensitivity and stability results for the nonlinear complementarity problem and variational inequalities. Meggido [30], Tobin [35] and Kyparisis [26] obtained some results on continuity of a locally unique solution $x(\varepsilon)$ of the parametric nonlinear complementarity problem $\operatorname{NCP}(F(\cdot, \varepsilon))$. All these papers assume differentiability of the function $F$ and they employ either the Implicit Function Theorem or the theory of generalized equations. Ha [18] used degree theory to derive sufficient conditions for the existence of solutions for small perturbations of the function. His approach does not require that the function $F$ be differentiable. Dafermos [5] employed an approach based on geometric arguments considering the variational inequalities as an orthogonal projection on some set. Facchinei and Pang [7] studied the "total stability" of the variational inequality $\operatorname{VIP}(K, F)$ when both the function $F$ and the set $K$ are perturbed. They employed degree theory and variational analytic tools to establish a general stability result for a variational inequality with a bounded solution set.

Our approach is totally different from theirs; we use tools of variational convergence and asymptotic analysis. We approximate the problem $\operatorname{VIP}\left(\mathbb{R}_{+}^{n}, F\right)$ by a sequence of problems $\operatorname{VIP}\left(D_{k}, F^{k}\right)(k \in \mathbb{N})$ where $\left\{D_{k}\right\}$ is a sequence of nested compact convex sets converging to $\mathbb{R}_{+}^{n}$ and $\left\{F^{k}\right\}$ is a sequence of continuous functions converging continuously to $F$. This approach allows us to develop a general theory yielding simultaneously existence, sensitivity and stability results. It is worth pointing out that our results are concerned with global sensitivity/stability analysis, which refers to the investigation of the change of the entire solution set SOL $(F)$ when the function $F$ undergoes small perturbation, and not with isolated sensitivity analysis as in most of the papers cited before.

This paper is a continuation of the previous work [11] where this new approach was employed for studying the multivalued complementarity problem. This approach has been developed when studying (LCP) in [9]. The novelty of the present paper with respect to the former lies in the fact that we now deal with single-valued mappings and to this end we employ another metric instead of that characterizing the graphical convergence (see also Rem. 1.9). The choice of this metric is natural and has the advantage that for the homogeneous type case it has an equivalent metric that allows us to obtain Lipschitzian properties for the solution-set-mapping for the piecewise affine case.

In Section 2, we list some preliminaries and introduce new classes of functions and the metrics we shall work with. In Section 3, we perform an asymptotic analysis of a sequence of normalized approximate solutions to (VIP) and obtain some bounds for the asymptotic set to the solution set. In Section 4, we study the nonemptiness and boundedness of the solution set for the new classes of functions and for the pseudomonotone ones. We organize, extend, and generalize most of the existing results from the literature. In Section 5, we obtain new sensitivity and stability results, study topological properties of the solution-set-mapping, and obtain some bounds for the solution set. Section 6 is devoted to study the piecewise affine case.

We shall use the following notation: $x \geq 0$ (resp. $x>0$ ) whenever $x \in \mathbb{R}_{+}^{n}$ (resp. $x \in$ int $\mathbb{R}_{+}^{n}$ ); $\mathbb{B}$ is the unit ball in $\mathbb{R}^{n} ; I=\{1, \ldots, n\} ;\|y\|_{\infty}$-maximum norm, $|y|=\left(\left|y_{1}\right|, \ldots,\left|y_{n}\right|\right),\|y\|_{d}=\langle d,|y|\rangle$ is the $d$-norm, $d_{\text {min }}=\min _{i \in I} d_{i}, \Delta_{d}=\left\{x \geq 0:\|x\|_{d}=1\right\}$ whenever $y \in \mathbb{R}^{n}$ and $d>0 ;\left\{e^{i}: i \in I\right\}$ is the canonical basis of $\mathbb{R}^{n}$; $\operatorname{SOL}(F)\left(\right.$ resp. $\left.\operatorname{FEA}(F)=\{x \geq 0: F(x) \geq 0\}, \operatorname{FEA}_{s}(F)=\{x \geq 0: F(x)>0\}\right)$ is the solution (resp. feasibility, strictly feasibility) set of $\operatorname{NCP}(F)$; given $d>0$ the set $A_{d}^{\infty}=\left\{v \in \mathbb{R}^{n}: \exists x^{k} \in A,\left\|x^{k}\right\|_{d} \rightarrow+\infty, \frac{x^{k}}{\left\|x^{k}\right\|_{d}} \rightarrow v\right\}$ is the $d$-asymptotic set of $A ; \mathrm{d}(x, A)$ is the distance of a point $x$ to $A ; \mathrm{d}(A, B)$ is the integrated set distance between $A$ and $B ; \mathcal{C}=\left\{c: \mathbb{R}_{++} \rightarrow \mathbb{R}_{++}: c(0) \geq 0, \lim _{t \rightarrow+\infty} c(t)=+\infty\right\}$.

In what follows, by $d$ and $c$ we shall denote a positive vector and a function from $\mathcal{C}$.

\section{Preliminary facts}

Here and in the subsequent sections we shall deal with functions $F$ defined on $\mathbb{R}_{+}^{n}$. We recall some well-known definitions from the literature (see $[8,19,34,37])$. A function $F: \mathbb{R}_{+}^{n} \rightarrow \mathbb{R}^{n}$ is said to be:

- copositive if $\langle F(x)-F(0), x\rangle \geq 0 \quad \forall x \geq 0$;

- strictly copositive if $\langle F(x)-F(0), x\rangle>0 \quad \forall 0 \neq x \geq 0$;

- strongly copositive if $\exists \alpha>0$ such that $\langle F(x)-F(0), x\rangle \geq \alpha\|x\|^{2} \forall x \geq 0$; 
- monotone if $\langle F(x)-F(y), x-y\rangle \geq 0 \quad \forall x, y \geq 0$;

- strictly monotone if $\langle F(x)-F(y), x-y\rangle>0 \quad \forall x, y \geq 0, x \neq y$;

- strongly monotone if $\exists \alpha>0$ such that $\langle F(x)-F(y), x-y\rangle \geq \alpha\|x-y\|^{2} \forall x, y \geq 0$;

- pseudomonotone if $\langle F(x), y-x\rangle \geq 0 \Longrightarrow\langle F(y), y-x\rangle \geq 0 \quad \forall x, y \geq 0$;

- a $\mathrm{G}(d)$-function ( $d$-Garcia's function), or $F \in \mathrm{G}(d)$, if $\operatorname{SOL}(F-F(0)+\tau d)=\{0\} \quad \forall \tau>0$;

- an $\mathrm{R}(d)$-function (d-regular function), or $F \in \mathrm{R}(d)$, if $\operatorname{SOL}(F-F(0)+\tau d)=\{0\} \forall \tau \geq 0$;

- an $\mathrm{R}_{0}$-function, or $F \in \mathrm{R}_{0}$, if $\operatorname{SOL}(F-F(0))=\{0\}$;

- piecewise affine if $\mathbb{R}_{+}^{n}$ can be represented as the union of finitely many polyhedral sets, relative to each of which $F(x)$ is given by an expression of the form $M x+q$ for $M \in \mathbb{R}^{n \times n}$ and $q \in \mathbb{R}^{n}$;

- $\mathbb{R}_{+}^{n}$-convex if $F(\lambda x+(1-\lambda) y) \leq \lambda F(x)+(1-\lambda) F(y) \quad \forall x, y \geq 0, \forall \lambda \in[0,1]$

If the inverse sign holds, we say that $F$ is $\mathbb{R}_{+}^{n}-$ concave;

- a $\mathrm{Q}_{\mathrm{b}}$-function if $\operatorname{SOL}(F+q)$ is nonempty and compact for all $q \in \mathbb{R}^{n}$.

We also recall some definitions concerning multifunctions, we shall need in what follows (see $[1,17,20,33,34]$ ).

Let $X$ and $Y$ be two metric spaces. A multifunction or set-valued mapping $\Phi: X \rightrightarrows Y$ is said to be:

- $\left(X=\mathbb{R}^{l}, Y=\mathbb{R}^{m}\right)$ piecewise polyhedral if its graph defined by gph $\Phi=\{(x, y): x \in \operatorname{dom} \Phi, y \in \Phi(x)\}$ is expressible as the union of finitely many polyhedral sets;

- $\left(X=\mathbb{R}^{l}, Y=\mathbb{R}^{m}\right)$ locally upper Lipschitzian at $\bar{x}$ with modulus $\lambda$ or locally $\mathrm{UL}(\lambda)$ at $\bar{x}$ if there is a neighborhood $U$ of $\bar{x}$ such that $\Phi(x) \subseteq \Phi(\bar{x})+\lambda\|x-\bar{x}\| \mathbb{B}$ for all $x \in U$. If in addition, $\Phi(\bar{x}) \neq \emptyset$ then $\Phi$ is called calm at $\bar{x}$;

- $\left(X=\mathbb{R}^{l}, Y=\mathbb{R}^{m}\right)$ Lipschitzian if there exits a scalar $\lambda>0$ such that $\Phi(x) \subseteq \Phi(y)+\lambda\|x-y\| \mathbb{B}$ for all $x$ and $y$;

- outer semicontinuous (OSC) (or closed) at $x \in \operatorname{dom} \Phi$ if whenever a sequence $\left\{\left(x^{k}, y^{k}\right)\right\} \subseteq \operatorname{gph} \Phi$ converges to $(x, y)$ then $y \in \Phi(x)$;

- inner semicontinuous (ISC) (or open or lower semicontinuous (lsc)) at $x \in \operatorname{dom} \Phi$ if for any $y \in \Phi(x)$ and for any sequence $\left\{x^{k}\right\} \subseteq \operatorname{dom} \Phi$ such that $x^{k} \rightarrow x$ there exists a sequence $\left\{y^{k}\right\}$ such that $y^{k} \in \Phi\left(x^{k}\right)$ for all $k$ and $y^{k} \rightarrow y$;

- continuous at $x \in \operatorname{dom} \Phi$ if it is OSC and ISC at $x$.

We now introduce two new classes of functions which are the single-valued analogous to those defined in [11] for the multivalued complementarity problem (see (b) of Ex. 1.3 below). As will be shown in the next example, such classes encompass various classes of functions from the literature that are important in the complementarity problem theory.

Definition 1.1. A function $F: \mathbb{R}_{+}^{n} \rightarrow \mathbb{R}^{n}$ is said to be:

- c-homogeneous $\left(\right.$ on $\left.\Delta_{d}\right)$ if $F(\lambda x)-F(0)=c(\lambda)[F(x)-F(0)]$ for all $x \in \Delta_{d}$ and $\lambda>0$;

- c-Moré $\left(\right.$ on $\left.\Delta_{d}\right)$ if $\langle F(\lambda x)-F(0), x\rangle \geq c(\lambda)\langle F(x)-F(0), x\rangle$ for all $x \in \Delta_{d}$ and $\lambda>0$.

Remark 1.2. (a) It is important to point out that our notions of $c$-homogeneous on $\Delta_{d}$ and $c$-Moré on $\Delta_{d}$ functions are given on the compact set $\Delta_{d}$ instead of being given on the whole $\mathbb{R}_{+}^{n}$ as is done in the literature (see Ex. 1.3). Henceforth, we shall consider that all the functions are $c$-homogeneous and $c$-Moré on the set $\Delta_{d}$ unless otherwise is specially stated.

(b) If the function $F$ is: copositive type, monotone type, $\mathrm{G}(\mathrm{d}), \mathrm{R}(\mathrm{d}), \mathrm{R}_{0}$, piecewise-affine, $\mathrm{Q}_{\mathrm{b}}, \mathbb{R}_{+}^{n}$-convex, $\mathbb{R}_{+}^{n}$-concave, $c$-homogeneous, or $c$-Moré, then so too is $F+q$ for all $q \in \mathbb{R}^{n}$. This property does not hold for pseudomonotone functions as is shown in Example 1.7 below.

(c) We may impose that the relationships defining $c$-homogeneous or $c$-Moré functions hold for all $\lambda$ sufficiently large (see [37]) or for all $\lambda \geq 1$ (see [11]) respectively. It is important to point out that our existence results hold under those assumptions. However, for obtaining also bounds for the solution set, sensitivity and stability results in a unified manner, our definitions are more suitable.

Example 1.3. (a) A function $H: \mathbb{R}_{+}^{n} \rightarrow \mathbb{R}^{n}$ is said to be (positively) generalized homogeneous (see [37]), if for some $c$ the equality $H(\lambda x)=c(\lambda) H(x)$ holds for all $x \geq 0$ and $\lambda>0$. In particular, if $c(\lambda)=\lambda^{\gamma}$, then $H$ is said to be (positively) homogeneous of degree $\gamma>0$. For instance, the functions $H^{1}(x)=M x$, where $M \in \mathbb{R}^{n \times n}$; 
$H^{2}(x)=\left(h_{1}^{2}(x), \ldots, h_{n}^{2}(x)\right)^{\top}$, where $h_{i}^{2}(x)=\max \left\{\left\langle w_{i j}, x\right\rangle: j \in \Lambda_{i}\right\}$ with $w_{i j} \in \mathbb{R}^{n}$ and $\Lambda_{i}$ being a finite index set, are homogeneous of degree 1. The function $H^{3}(x)=\|x\| M x$ is homogeneous of degree 2.

If $F-F(0)$ is generalized homogeneous (for some $c$ ), then $F$ is $c$-homogeneous on $\Delta_{d}$ for any $d$. The functions $F^{1}(x)=x$ if $0 \leq x \leq 1$ and $F^{1}(x)=3 x-2$ if $x \geq 1, F^{2}(x)=x$ if $0 \leq x \leq 1$ and $F^{2}(x)=x^{2}$ if $x \geq 1$, and $F^{3}(x, y)=e^{x+y} \sqrt{x^{2}+y^{2}}(x, y)^{\top}$ are $c$-homogeneous on $\Delta_{d}$ for $d=1, c_{1}(\lambda)=F^{1}(\lambda), c_{2}(\lambda)=F^{2}(\lambda)$, and $d=(1,1)^{\top}, c_{3}(\lambda)=\lambda^{2} e^{\lambda-1}$ respectively. However, $F^{i}(x)-F^{i}(0)(i=1,2,3)$ are not generalized homogeneous. (b) A multifunction $\Phi: \mathbb{R}_{+}^{n} \rightrightarrows \mathbb{R}^{n}$ is said to be $c$-subhomogeneous, if $0 \in \Phi(0)$ and for some $c$ it holds that $\Phi(\lambda x) \subseteq c(\lambda) \Phi(x)$ for all $x \in \Delta_{d}$ and $\lambda>0$ (see [11]). If $\Phi$ is single-valued, then $\Phi$ is $c$-homogeneous. Therefore, for single-valued mappings the classes of $c$-subhomogeneous and $c$-homogeneous mappings coincide.

(c) A function $F: \mathbb{R}_{+}^{n} \rightarrow \mathbb{R}^{n}$ such that $\langle F(\lambda x)-F(0), x\rangle \geq c(\lambda)\langle F(x)-F(0), x\rangle$ for some $c$ and for all $x \geq 0$ and $\lambda>0$ (see [37]) is $c$-Moré on $\Delta_{d}$ for any $d$. For instance, $F$ such that $F(\lambda x)-F(0) \geq c(\lambda)[F(x)-F(0)]$ for some $c$ and for all $x \geq 0$ and $\lambda>0$ (see [31]) satisfies such a condition. The function $F(x, y)=\left(x^{3}, y^{4}\right)^{\top}$ satisfies the latter inequality for $\tilde{c}(\lambda)=\min \left\{\lambda^{3}, \lambda^{4}\right\}$ but it is not $c$-homogeneous on $\Delta_{d}$ for any $c$ and $d$.

(d) The functions $F^{4}(x)=M x+q$ for $M \in \mathbb{R}^{n \times n}, q \in \mathbb{R}^{n}$, and $F^{5}(x)=P_{C}(x)$-projection function onto the polyhedral set $C \subseteq \mathbb{R}^{n}$ are piecewise affine (see Ex. 12.31 from [34]). If $T: \mathbb{R}^{n} \rightrightarrows \mathbb{R}^{n}$ is maximal monotone piecewise polyhedral and $f: \mathbb{R}^{n} \rightarrow \mathbb{R}$ is proper lsc convex piecewise linear-quadratic, then the resolvent and proximal mappings $R_{\lambda}(x)=(I+\lambda T)^{-1}$ and $P_{\lambda} f(x)=\arg \min _{w}\left\{f(w)+\frac{1}{2 \lambda}\|w-x\|^{2}\right\}$ are piecewise affine for every $\lambda>0$ (see Props. 12.29 and 12.30 from [34] respectively).

(e) If $F$ is copositive (resp. strictly copositive), then $F \in \mathrm{G}(\mathrm{d})$ (resp. $F \in \mathrm{R}(\mathrm{d})$ ) for all $d$. Indeed, if $x \in \mathrm{SOL}(F-$ $F(0)+\tau d)$ for $\tau>0$ (resp. $\tau \geq 0$ ), then $0 \leq x \perp F(x)-F(0)+\tau d \geq 0$, therefore, $0 \leq\langle F(x)-F(0), x\rangle=-\tau\langle d, x\rangle$ a contradiction if $x \neq 0$.

The following definition generalizes that used for linear mappings in [21] and is the single-valued variant of that used for multifunctions in [11].

Definition 1.4. Let $F: \mathbb{R}_{+}^{n} \rightarrow \mathbb{R}^{n}$ be a continuous function. The $d$-numerical range of $F$ is by definition the set $\omega(F):=\left\{\langle F(x)-F(0), x\rangle: x \in \Delta_{d}\right\}$. We define $M_{F}:=\max \omega(F)$ and $m_{F}:=\min \omega(F)$.

The next result is similar to Propositions 2.5 and 4.5 from [11] and complements them. Part (a) generalizes Corollary 3.3 from [31].

Proposition 1.5. Let $F: \mathbb{R}_{+}^{n} \rightarrow \mathbb{R}^{n}$ be a continuous function.

(a) if $F$ is c-Moré, then $m_{F}\|x\|_{d} c\left(\|x\|_{d}\right) \leq\langle F(x)-F(0)$, $x\rangle$ for all $x \geq 0$;

(b) if $F$ is c-homogeneous, then $F$ is c-Moré and $\langle F(x)-F(0), x\rangle \leq M_{F}\|x\|_{d} c\left(\|x\|_{d}\right)$ for all $x \geq 0$;

(c) if $F$ is strongly copositive, then $F$ is $\bar{c}$-Moré on $\Delta_{d}$ for any $d$ and $\bar{c}(\lambda)=\frac{\alpha \lambda}{M_{F}\|d\|^{2}}$;

(d) if $F$ is strictly copositive, then $m_{F}>0$. If in addition, $F$ is homogeneous of degree 1 , then $F$ is strongly copositive;

(e) if $F$ is c-Moré and $m_{F}>0$, then $F$ is strictly copositive;

(f) if $F$ is $\mathbb{R}_{+}^{n}$-convex, then it holds that $F(\lambda x)-F(0) \geq \lambda[F(x)-F(0)]$ for all $x \geq 0$ and $\lambda \geq 1$, and $F(\lambda x)-F(0) \leq \lambda[F(x)-F(0)]$ for all $x \geq 0$ and $\lambda \in[0,1]$;

if in addition, $F$ is strictly copositive, then $F$ is $\tilde{c}$-Moré on $\Delta_{d}$ for any $d$ and $\tilde{c}(\lambda)=\lambda$ if $\lambda \geq 1$ and $\tilde{c}(\lambda)=\min _{x \in \Delta_{d}} \frac{\langle F(\lambda x)-F(0), x\rangle}{\langle F(x)-F(0), x\rangle}$ if $0 \leq \lambda \leq 1 ;$

(g) if $F$ is pseudomonotone c-homogeneous and $\mathrm{FEA}(F) \neq 0$, then $F$ is copositive.

Proof. (a)-(b): Are trivial.

(c): If $x \in \Delta_{d}$ and $\lambda>0$, then by hypothesis and since it holds that $1=\|x\|_{d} \leq\|d\|\|x\|$ we obtain $\langle F(\lambda x)-F(0), \lambda x\rangle \geq \alpha \lambda^{2}\|x\|^{2} \geq \frac{\alpha \lambda^{2}}{\left.\|d\|\right|^{2} M_{F}}\langle F(x)-F(0), x\rangle$.

(d): If $F$ is strictly copositive, then by Weierstrass theorem $m_{F}>0$. Moreover, by the homogeneity of degree 1 and (a), we obtain $\langle F(x)-F(0), x\rangle \geq m_{F}\|x\|_{d}^{2} \geq m_{F} d_{\min }^{2}\|x\|^{2}$ for all $0 \neq x \geq 0$.

(e): It follows from (a).

(f): If $x \geq 0$, then by hypothesis $F(x)=F\left(\frac{1}{\lambda}(\lambda x)+\left(1-\frac{1}{\lambda}\right) 0\right) \leq \frac{1}{\lambda} F(\lambda x)+\left(1-\frac{1}{\lambda}\right) F(0)$ for $\lambda \geq 1$, thus, 
$F(\lambda x)-F(0) \geq \lambda[F(x)-F(0)]$. The proof for $\lambda \in[0,1]$ runs as before. The rest of the proof is immediate. (g): If $\bar{x} \in \operatorname{FEA}(F)$ and $x>0$, then there exists $t_{x}>0$ such that $\frac{t}{\|x\|_{d}} x-\bar{x} \geq 0$ and $\left\langle F(\bar{x}), \frac{t}{\|x\|_{d}} x-\bar{x}\right\rangle \geq 0$ for all $t>t_{x}$. By $c$-homogeneity we have $F\left(\frac{t}{\|x\|_{d}} x\right)-F(0)=\frac{c(t)}{c\left(\|x\|_{d}\right)}[F(x)-F(0)]$ and by using the last inequality and the pseudomonotonicity hypothesis we conclude that $\left\langle F(0)+\frac{c(t)}{c\left(\|x\|_{d}\right)}[F(x)-F(0)], \frac{t}{\|x\|_{d}} x-\bar{x}\right\rangle \geq 0$. On dividing the previous inequality by $c(t) t$ and taking the limit $t \rightarrow+\infty$, we get $\langle F(x)-F(0), x\rangle \geq 0$ for all $x>0$. If $x$ lies on the boundary of $\mathbb{R}_{+}^{n}$, then there exists $\left\{x^{k}\right\} \subseteq \operatorname{int} \mathbb{R}_{+}^{n}$ such that $x^{k} \rightarrow x$ and $\left\langle F\left(x^{k}\right)-F(0), x^{k}\right\rangle \geq 0$. By the continuity of $F$ we obtain $\langle F(x)-F(0), x\rangle \geq 0$.

Our approach consists of the approximation of (VIP) by a sequence of solvable problems and the later study of the asymptotic properties of a sequence of normalized solutions of such approximations. To do that we must introduce a suitable metric space.

The Metric SPACE $\left(\mathrm{C}\left(\mathbb{R}_{+}^{\mathbf{n}} ; \mathbb{R}^{\mathbf{n}}\right), \mathbb{D}\right)$ : where $\mathrm{C}\left(\mathbb{R}_{+}^{n} ; \mathbb{R}^{n}\right)$ is the set of continuous functions $F=\left(f_{1}, \ldots, f_{n}\right)^{\top}$ defined on $\mathbb{R}_{+}^{n}$ and $\mathbb{D}$ is the metric defined by:

$$
\mathbb{D}(F, G):=\max _{i \in I} \mathbb{I}_{\mathrm{epi}}\left(f_{i}, g_{i}\right)+\max _{i \in I} \mathbb{I}_{\mathrm{hyp}}\left(f_{i}, g_{i}\right)
$$

where $\mathrm{dI}_{\mathrm{epi}}$ and $\mathrm{dI}_{\mathrm{hyp}}$ are the metrics which characterize the epi-convergence $(\stackrel{e}{\rightarrow})$ and hypo-convergence $(\stackrel{h}{\rightarrow})$ respectively and for $f, g \in \mathrm{C}\left(\mathbb{R}_{+}^{n} ; \mathbb{R}\right)$ are defined by dI $\mathbb{L}_{\text {epi }}(f, g):=\mathrm{dI}($ epi $f$, epi $g)$ and $\mathbb{d I}_{\text {hyp }}(f, g):=\operatorname{dI}($ hyp $f$, hyp $g)$ where the right-hand terms are the integrated set distances between the epigraphs and hypographs of $f$ and $g$ respectively (see Chaps. 5 and 7 from [34]).

In order to obtain some properties for this metric, we recall a well-known type of convergence. A sequence of functions $\left\{F^{k}\right\}$ from $\mathbb{R}^{n}$ to $\mathbb{R}^{n}$ converges continuously to the function $F$ if $F^{k}\left(x^{k}\right) \rightarrow F(x)$ whenever $x^{k} \rightarrow x$. In Proposition 7.2 from [34] it is proved that for $\left\{f^{k}\right\}$ and $f$ being functions from $\mathbb{R}^{n}$ to $\mathbb{R}$ it holds that:

- $f^{k} \stackrel{e}{\rightarrow} f$ if and only if at each point $x$ one has:

$\left\{\liminf _{k} f^{k}\left(x^{k}\right) \geq f(x)\right.$ for every sequence $x^{k} \rightarrow x$

$\left\{\limsup _{k} f^{k}\left(x^{k}\right) \leq f(x)\right.$ for some sequence $x^{k} \rightarrow x$
- $f^{k} \stackrel{h}{\rightarrow} f$ if and only if at each point $x$ one has: $\left\{\begin{aligned} \liminf _{k} f^{k}\left(x^{k}\right) & \geq f(x) \text { for some sequence } x^{k} \rightarrow x \\ \lim \sup _{k} f^{k}\left(x^{k}\right) & \leq f(x) \text { for every sequence } x^{k} \rightarrow x .\end{aligned}\right.$

Thus, by joining both assertions we conclude that $f^{k} \stackrel{e}{\rightarrow} f$ and $f^{k} \stackrel{h}{\rightarrow} f$ iff $\left\{f^{k}\right\}$ converges continuously to $f$ (see Th. 7.11 from [34]). Consequently, $\mathbb{D}\left(F^{k}, F\right) \rightarrow 0$ iff $\left\{F^{k}\right\}$ converges continuously to $F$.

Proposition 1.6. Let $\left\{F^{k}\right\}$ and $F$ be functions such that $\mathbb{D}\left(F^{k}, F\right) \rightarrow 0$. If the sequence $\left\{F^{k}\right\}$ is from one of the following classes: c-homogeneous, c-Moré, copositive, or monotone, then so too is $F$.

Proof. Let $\left\{F^{k}\right\}$ be from the first class of functions and let $x \in \Delta_{d}$ and $\lambda>0$ be given. By hypothesis we have $F^{k}(\lambda x)-F^{k}(0)=c(\lambda)\left[F^{k}(x)-F^{k}(0)\right]$. Taking limit and by the continuous convergence of $\left\{F^{k}\right\}$ to $F$ we get $F(\lambda x)-F(0)=c(\lambda)[F(x)-F(0)]$, thus, $F$ is $c$-homogeneous. The rest of the proof runs as before.

Example 1.7. Each function $F^{k}(x, y)=\left(y+\frac{1}{k}, 0\right)^{\top}$ is pseudomonotone and $\mathbb{D}\left(F^{k}, F\right) \rightarrow 0$ for $F(x, y)=(y, 0)^{\top}$, but $F$ is not pseudomonotone since $\left\langle F\left(e^{1}\right), e^{2}-e^{1}\right\rangle=0$ and $\left\langle F\left(e^{2}\right), e^{2}-e^{1}\right\rangle<0$.

We now introduce a new metric space, which plays an important role when dealing with $c$-homogeneous functions.

The METRIC SPACE $\left(\mathrm{C}\left(\mathbb{R}_{+}^{\mathbf{n}} ; \mathbb{R}^{\mathbf{n}}\right)_{\mathbf{c}}, \mathbb{D}_{\mathrm{o}}\right)$ : where $\mathrm{C}\left(\mathbb{R}_{+}^{n} ; \mathbb{R}^{n}\right)_{c}$ is the set of $c$-homogeneous continuous functions $F=\left(f_{1}, \ldots, f_{n}\right)^{\top}$ defined on $\mathbb{R}_{+}^{n}$ and $\mathbb{D}_{\mathrm{o}}$ is the metric defined by:

$$
\mathbb{D}_{\mathrm{o}}(F, H):=\max _{x \in \Delta_{d}}\|F(x)-F(0)-H(x)+H(0)\|_{\infty}+\|F(0)-H(0)\|_{\infty}
$$

It is not difficult to prove that $\mathbb{D}_{o}\left(F^{k}, F\right) \rightarrow 0$ iff $\left\{F^{k}\right\}$ converges uniformly to $F$ on $\Delta_{d}$ and $F^{k}(0) \rightarrow F(0)$. 


\section{Proposition 1.8.}

(a) If $F, H \in \mathrm{C}\left(\mathbb{R}_{+}^{n} ; \mathbb{R}\right)_{c}^{n}$, then $\|F(x)-H(x)\|_{\infty} \leq c\left(\|x\|_{d}\right) \mathbb{D}_{o}(F, H)+\|F(0)-H(0)\|_{\infty}$ for all $x \geq 0$;

(b) if $c$ is nondecreasing and $\left\{F^{k}\right\}, F$ are from $\mathrm{C}\left(\mathbb{R}_{+}^{n} ; \mathbb{R}^{n}\right)_{c}$, then $\mathbb{D}_{\mathrm{o}}\left(F^{k}, F\right) \rightarrow 0$ iff $\mathbb{D}\left(F^{k}, F\right) \rightarrow 0$. Hence, $\mathbb{D}$ and $\mathbb{D}_{\mathrm{o}}$ are equivalent metrics on $\mathrm{C}\left(\mathbb{R}_{+}^{n} ; \mathbb{R}^{n}\right)_{c}$.

Proof. (a): For $x=0$ the assertion is trivial. If $0 \neq x \geq 0$, then by the $c$-homogeneity we have

$\|F(x)-H(x)\|_{\infty} \leq\|F(x)-F(0)-[H(x)-H(0)]\|_{\infty}+\|F(0)-H(0)\|_{\infty} \leq c\left(\|x\|_{d}\right) \mathbb{D}_{\mathrm{o}}(F, H)+\|F(0)-H(0)\|_{\infty}$.

(b): "Only if part": If $\left\{x^{k}\right\}$ converges to $x$, then there exists an $r>0$ such that $\left\|x^{k}\right\|_{d} \leq r$ for all $k$, thus, by hypothesis $c\left(\left\|x^{k}\right\|_{d}\right) \leq c(r)$. By (a), the continuity of $F$, and the following inequalities

$$
\begin{aligned}
\left\|F^{k}\left(x^{k}\right)-F(x)\right\|_{\infty} & \leq\left\|F^{k}\left(x^{k}\right)-F\left(x^{k}\right)\right\|_{\infty}+\left\|F\left(x^{k}\right)-F(x)\right\|_{\infty} \\
& \leq c(r) \mathbb{D}_{0}\left(F^{k}, F\right)+\left\|F^{k}(0)-F(0)\right\|_{\infty}+\left\|F\left(x^{k}\right)-F(x)\right\|_{\infty}
\end{aligned}
$$

we conclude that $F^{k}\left(x^{k}\right) \rightarrow F\left(x^{k}\right)$. Hence $\mathbb{D}\left(F^{k}, F\right) \rightarrow 0$.

"If part": The hypothesis implies that $\left\{F^{k}\right\}$ converges uniformly to $F$ on all compact subsets of $\mathbb{R}_{+}^{n}$ (see Th. 7.14 from [34]). In particular, this holds on $\Delta_{d}$ and $\{0\}$. Hence $\mathbb{D}_{\mathrm{o}}\left(F^{k}, F\right) \rightarrow 0$.

The last part follows from the fact that two metrics are equivalent iff they induce the same convergence.

Remark 1.9. By Corollary 5.45 from [34] it is known that $\left\{F^{k}\right\}$ converges continuously to $F$ iff $\left\{F^{k}\right\}$ converges graphically to $F$ and the sequence $\left\{F^{k}\right\}$ is eventually locally bounded at each $\bar{x}$, i.e., there exist a neighborhood $V$ of $\bar{x}, k_{0} \in \mathbb{N}$, and a bounded set $B$ such that $F^{k}(V) \in B$ for all $k \geq k_{0}$. Therefore, if we approximate (VIP) by using the continuous convergence, then the approximation is also by using the graphical convergence and the results from [11] hold (in terms of the metric characterizing the graphical convergence). However, if we approximate (VIP) by using the graphical convergence we cannot capture all the results of this paper (unless an additional assumption is assumed).

In the next three sections we shall employ the metric $\mathbb{D}$ for obtaining our results. In the last section we shall employ the equivalent metric $\mathbb{D}_{\mathrm{o}}$ to deal with $c$-homogeneous piecewise affine functions for $c$ being nondecreasing.

\section{Asymptotic AnALYSis}

We approximate (VIP) by the following sequence of problems:

$$
\text { find } x^{k} \in D_{k}:\left\langle F^{k}\left(x^{k}\right), x-x^{k}\right\rangle \geq 0 \quad \forall x \in D_{k}
$$

where $d>0,\left\{\sigma_{k}\right\}$ is an increasing sequence of positive numbers converging to $+\infty, D_{k}=\left\{x \in \mathbb{R}_{+}^{n}:\|x\|_{d} \leq \sigma_{k}\right\}$ and $\mathbb{D}\left(F^{k}, F\right) \rightarrow 0$.

The existence of solutions $x^{k}$ to this problem is a consequence of the following result (see [25]).

Theorem 2.1 (Hartman-Stampacchia). Let $C \subset \mathbb{R}^{n}$ be a compact convex nonempty set and let $F: C \rightarrow \mathbb{R}^{n}$ be a continuous function. There exists a vector $\bar{x} \in C$ such that $\langle F(\bar{x}), x-\bar{x}\rangle \geq 0$ for all $x \in C$.

Henceforth, we assume that all the functions we shall deal with are from $\mathrm{C}\left(\mathbb{R}_{+}^{n} ; \mathbb{R}^{n}\right)$.

We now introduce some sets called coercive existence sets, which are fundamental in our study. The importance of these sets lies in the fact that they provide valuable information on $(\mathrm{NCP})$ : the accumulation points of any sequence of normalized approximate solutions belong to these sets (see Lem. 2.3), they bound the asymptotic cones/sets of the solution set (see Cor. 2.4), and our main existence, stability, and sensitivity results 
are given in terms of a property satisfied by these sets (the property of being equal to $\{0\}$ ):

$$
\begin{aligned}
\mathrm{W}(F) & =\left\{v \geq 0: \tau_{v}=-\langle F(v)-F(0), v\rangle \geq 0, F(v)-F(0)+\tau_{v} d \geq 0\right\} \\
\mathrm{W}_{q}(H) & =\{v \geq 0: H(v)-H(0) \geq 0,\langle H(v)-H(0), v\rangle=0,\langle H(0)+q, v\rangle \leq 0\} \\
\mathrm{U}(F) & =\{v \geq 0:\langle F(v)-F(0), v\rangle \leq 0\} \\
\mathrm{U}_{q}(H) & =\{v \geq 0:\langle H(v)-H(0), v\rangle=0,\langle H(0)+q, v\rangle \leq 0\} \\
\mathrm{V}(F) & =\mathbb{R}_{+}^{n} \cap\left[-F\left(\mathbb{R}_{+}^{n}\right)\right]^{*} .
\end{aligned}
$$

Two of these sets can be written by means of the set $\mathrm{SOL}(H-H(0))$, termed complementary kernel of $\mathrm{NCP}(H)$.

$$
\mathrm{W}_{q}(H)=\operatorname{SOL}(H-H(0)) \cap\{-H(0)-q\}^{*} \text { and } \mathrm{W}_{-H(0)}(H)=\mathrm{SOL}(H-H(0))
$$

and if $\tau_{v}=-\langle F(v)-F(0), v\rangle \geq 0$ as above, then

$$
\left[v \in \mathrm{W}(F) \text { and }\|v\|_{d}=1\right] \Longrightarrow v \in \operatorname{SOL}\left(F-F(0)+\tau_{v} d\right) .
$$

\section{Proposition 2.2.}

(a) if $F-F(0)$ is homogeneous of degree $\gamma>0$, then $\mathrm{SOL}(F-F(0))^{\#}=\operatorname{int} \mathrm{SOL}(F-F(0))^{*}$. Moreover, $q \in \operatorname{int} \mathrm{SOL}(F-F(0))^{*}$ iff $\mathrm{W}_{q}(F)=\{0\}$

(b) if $\mathrm{FEA}_{s}(F) \neq \emptyset$, then $\mathrm{V}(F)=\{0\}$. The inverse implication holds if $F$ is pseudomonotone;

(c) if $F$ is copositive, then $\mathrm{U}_{-F(0)}(F)=\mathrm{U}(F)$. Moreover, $F(0)+q \in \mathrm{U}(F)^{\#}$ iff $\mathrm{U}_{q}(F)=\{0\}$;

(d) if $F$ is c-homogeneous, then $\mathrm{SOL}(F-F(0))$ is a cone.

Proof. (a): The equality follows from Exercise 6.22 of [34] and the remainder is obvious.

(b): If $\bar{x} \in \mathrm{FEA}_{s}(F)$ and $v \in \mathrm{V}(F)$, then $\langle F(\bar{x}), v\rangle \leq 0$, a contradiction if $v \neq 0$. For the inverse implication see Theorem 2.4.4 from [8].

(c): It is obvious.

(d): Let $0 \neq x \in \operatorname{SOL}(F-F(0))$ be given. By hypothesis $\frac{c\left(t|| x \mid \|_{d}\right)}{c\left(\|x\|_{d}\right)}[F(x)-F(0)]=F(t x)-F(0)$ for all $t>0$. Multiplying $F(x)-F(0) \geq 0$ and $\langle F(x)-F(0), x\rangle=0$ by $\frac{c\left(t|| x \mid \|_{d}\right)}{c\left(|x| \|_{d}\right)}$ and $\frac{c\left(t|| x \mid \|_{d}\right) t}{c\left(\|x\|_{d}\right)}$ respectively, we obtain that $t x \in \operatorname{SOL}(F-F(0))$ for all $t>0$.

Lemma 2.3 (Basic Lemma). Let $\left\{x^{k}\right\}$ be a sequence of solutions to $\left(\mathrm{PVIP}_{\mathrm{k}}\right)$ such that $\left\|x^{k}\right\|_{d}=\sigma_{k}$ and $\frac{x^{k}}{\sigma_{k}} \rightarrow v$.

(a) If each $F^{k}$ is c-homogeneous, then $v \in \mathrm{W}(F) \cap \mathrm{SOL}\left(F-F(0)+\tau_{v} d\right) \cap \Delta_{d}$.

(i) If $F$ is also a $\mathrm{G}(d)$-function, then $v \in \mathrm{SOL}(F-F(0)) \cap \Delta_{d}$;

(ii) if each $F^{k}=H^{k}+q^{k}$ is also copositive and $F(x)=H+q$, then $v \in \mathrm{W}_{q}(H) \cap \Delta_{d}$;

(b) if each $F^{k}$ is c-Moré, then $v \in \mathrm{U}(F) \cap \Delta_{d}$.

If each $F^{k}=H^{k}+q^{k}$ is also copositive and $F=H+q$, then $v \in \mathrm{U}_{q}(H) \cap \Delta_{d}$;

(c) if each $F^{k}$ is pseudomonotone, then $v \in \mathrm{V}(F) \cap \Delta_{d}$.

Proof. (a): By hypothesis $F^{k}\left(x^{k}\right)-F^{k}(0)=c\left(\sigma_{k}\right)\left[F^{k}\left(\frac{x^{k}}{\sigma_{k}}\right)-F^{k}(0)\right]$. Replacing this in $\left(\mathrm{PVIP}_{\mathrm{k}}\right)$, dividing by $c\left(\sigma_{k}\right) \sigma_{k}$, and taking limit for $x=0$ and $x=\sigma_{k} \frac{y}{\|y\|_{d}}$ with $0 \neq y \geq 0$ respectively, by the continuous convergence of $\left\{F^{k}\right\}$ to $F$ we obtain $\langle F(v)-F(0), v\rangle \leq 0$ and $\langle F(v)-F(0), y\rangle \geq\|y\|_{d}\langle F(v)-F(0), v\rangle$ for all $y \geq 0$. By taking $y=e^{i}$ for $i \in I$, we get $v \in \mathrm{W}(F)$ and by (2.2) we conclude that $v \in \operatorname{SOL}\left(F-F(0)+\tau_{v} d\right)$. (i): If $F \in \mathrm{G}(\mathrm{d})$, then necessarily $\tau_{v}=0$.

(ii): If each $F^{k}=H^{k}+q^{k}$ is copositive, then so too are $H^{k}$ and $H$ (see Prop. 1.6). Clearly, $H \in \mathrm{G}(\mathrm{d})$ and by (i) we get $\tau_{v}=0$, thus, $0 \leq v \perp H(v)-H(0) \geq 0$. By taking $x=0$ in $\left(\mathrm{PVIP}_{k}\right)$ and by the copositivity of $H^{k}$ we obtain $0 \geq\left\langle H^{k}\left(x^{k}\right)+q^{k}, x^{k}\right\rangle=\left\langle H^{k}\left(x^{k}\right)-H^{k}(0), x^{k}\right\rangle+\left\langle H^{k}(0)+q^{k}, x^{k}\right\rangle \geq\left\langle H^{k}(0)+q^{k}, x^{k}\right\rangle$. On dividing by $\sigma_{k}$ and taking limit we obtain $\langle H(0)+q, v\rangle \leq 0$.

(b): By hypothesis we have $\left\langle F^{k}\left(x^{k}\right)-F^{k}(0), x^{k}\right\rangle \geq c\left(\sigma_{k}\right)\left\langle F^{k}\left(\frac{x^{k}}{\sigma_{k}}\right)-F^{k}(0), x^{k}\right\rangle$. Replacing this inequality 
in $\left(\mathrm{PVIP}_{\mathrm{k}}\right)$ for $x=0$ we get $0 \geq\left\langle F^{k}\left(x^{k}\right)-F^{k}(0), x^{k}\right\rangle+\left\langle F^{k}(0), x^{k}\right\rangle \geq c\left(\sigma_{k}\right)\left\langle F^{k}\left(\frac{x^{k}}{\sigma_{k}}\right)-F^{k}(0), x^{k}\right\rangle+\left\langle F^{k}(0), x^{k}\right\rangle$. On dividing by $c\left(\sigma_{k}\right) \sigma_{k}$ and taking limit, by the continuous convergence of $\left\{F^{k}\right\}$ to $F$ we get $\langle F(v)-F(0), v\rangle \leq 0$.

If each $F^{k}=H^{k}+q^{k}$ is copositive, then we proceed similarly as in (a).

(c): If $x \geq 0$ is arbitrary, then there exists $k_{x} \in \mathbb{N}$ such that $\left(\mathrm{PVIP}_{\mathrm{k}}\right)$ holds for all $k \geq k_{x}$ and by hypothesis we get $\left\langle F^{k}(x), x-x^{k}\right\rangle \geq 0$. Dividing by $\sigma_{k}$ and taking limit, by the continuous convergence of $\left\{F^{k}\right\}$ to $F$ we obtain $\langle F(x), v\rangle \leq 0$.

We can characterize the boundedness of a nonempty set $A \subseteq \mathbb{R}^{n}$ by means of its asymptotic cone $A^{\infty}$ or its $d$-normalized asymptotic set $A_{d}^{\infty}$ as follows: $A$ is bounded iff $\bar{A}^{\infty}=\{0\}$ iff $A_{d}^{\infty}=\emptyset$ (see $[2,11]$ ). Therefore, for studying the boundedness of the solution set to (NCP) we now obtain bounds for the asymptotic sets/cones of this set. These bounds are related to those for affine variational inequality problems from $[8,32]$ and for multivalued complementarity problems from [11].

\section{Corollary 2.4.}

(a) For $H$ being c-homogeneous: $\bigcup_{q \in \mathbb{R}^{n}} \operatorname{SOL}(H+q)^{\infty} \subseteq \operatorname{SOL}(H-H(0))$. If in addition, $H$ is copositive, then $\mathrm{SOL}(H+q)^{\infty} \subseteq \mathrm{W}_{q}(H)$.

(b) For $H$ being c-Moré: $\bigcup_{q \in \mathbb{R}^{n}} \operatorname{SOL}(H+q)_{d}^{\infty} \subseteq \mathrm{U}(H) \cap \bar{\Delta}_{d}$. If in addition, $H$ is copositive, then $\mathrm{SOL}(H+q)_{d}^{\infty} \subseteq \mathrm{U}_{q}(H) \cap \Delta_{d}$.

(c) If $F$ is pseudomonotone and $\operatorname{SOL}(F) \neq \emptyset$, then $\operatorname{SOL}(F)^{\infty}=\mathrm{V}(F)$.

Proof. (a): Let $q \in \mathbb{R}^{n}$ be fixed and $v \in \operatorname{SOL}(H+q)^{\infty}$. If $v=0$, the assertion is trivial. If $v \neq 0$, there exists $\left\{x^{k}\right\} \subseteq \operatorname{SOL}(H+q)$ and $t_{k} \downarrow 0$ such that $t_{k} x^{k} \rightarrow v$. Clearly, $H\left(x^{k}\right)-H(0)=c\left(\left\|x^{k}\right\|_{d}\right)\left[H\left(\frac{t_{k} x^{k}}{\left\|t_{k} x^{k}\right\|_{d}}\right)-H(0)\right]$. By replacing this equality in $H\left(x^{k}\right)+q \geq 0$ and $\left\langle H\left(x^{k}\right)+q, x^{k}\right\rangle=0$, dividing by $c\left(\left\|x^{k}\right\|_{d}\right)$ and $c\left(\left\|x^{k}\right\|_{d}\right)\left\|x^{k}\right\|_{d}$ respectively, and taking limit we obtain $0 \leq \frac{v}{\|v\|_{d}} \perp H\left(\frac{v}{\|v\|_{d}}\right)-H(0) \geq 0$. By Proposition 2.2(d) we conclude that $v \in \mathrm{SOL}(H-H(0))$.

If $H$ is also copositive, from $0=\left\langle H\left(x^{k}\right)-H(0), x^{k}\right\rangle+\left\langle H(0)+q, x^{k}\right\rangle \geq\left\langle H(0)+q, x^{k}\right\rangle$ we conclude that $\langle H(0)+q, v\rangle \leq 0$.

(b): Let $q \in \mathbb{R}^{n}$ be fixed and $v \in \operatorname{SOL}(H+q)_{d}^{\infty}$. There exists a sequence $\left\{x^{k}\right\}$ such that $\left\|x^{k}\right\|_{d} \rightarrow+\infty$ and $\frac{x^{k}}{\left\|x^{k}\right\|_{d}} \rightarrow v$. By setting $\sigma_{k}:=\left\|x^{k}\right\|_{d}$ and since each $x^{k}$ is solution to $\left(\mathrm{PVIP}_{k}\right)$ for $F^{k}=H+q$ for all $k$, by the first part of (b) of the Basic Lemma for such $F^{k}$, we conclude that $v \in \mathrm{U}(H) \cap \Delta_{d}$.

If $H$ is also copositive, then the second bound follows by using the last part of (b) of the Basic Lemma.

(c): If $F$ is pseudomonotone, then it is well-known that (see Lem. 2.1 from [22])

$$
\operatorname{SOL}(F)=\bigcap_{x \geq 0}\{\bar{x} \geq 0:\langle F(x), x-\bar{x}\rangle \geq 0\}
$$

Since each right-hand set is closed and convex and $\mathrm{SOL}(F) \neq \emptyset$, by properties of asymptotic cones (see Props. 3.9 and 3.23 from [34]) we conclude that $\operatorname{SOL}(F)^{\infty}=\bigcap_{x \geq 0}\{\bar{x} \geq 0:\langle F(x), x-\bar{x}\rangle \geq 0\}^{\infty}=\mathrm{V}(F)$.

\section{Main EXistence Results}

In this section, we obtain coercive existence results for $(\mathrm{NCP})$. To do this, in $\left(\mathrm{PVIP}_{k}\right)$ we consider that $F^{k}=F$ for all $k$, i.e., we approximate (VIP) by the following sequence of problems

$$
\text { find } x^{k} \in D_{k}:\left\langle F\left(x^{k}\right), x-x^{k}\right\rangle \geq 0 \forall x \in D_{k} .
$$

We now recall an existence theorem for (VIP), which is related to this approximation (see Th. 4.2 from [25]). We give the proof for reader's convenience.

Proposition 3.1 (Hartman-Stampacchia). A necessary and sufficient condition that there exist a solution to (VIP) is that there exists a number $k$ such that $\left\|x^{k}\right\|_{d}<\sigma_{k}$. 
Proof. If there exists a solution $x$ to (VIP), then $x$ is a solution to $\left(\mathrm{VIP}_{k}\right)$ whenever $\|x\|_{d}<\sigma_{k}$.

If we suppose that $\left\|x^{k}\right\|_{d}<\sigma_{k}$ for some $k$, then $x^{k}$ is also a solution to (VIP). Indeed, given $x \in \mathbb{R}_{+}^{n}$, we get $w=x^{k}+\varepsilon\left(x-x^{k}\right) \in D_{k}$ for $\varepsilon>0$ sufficiently small. Thus, $0 \leq\left\langle F\left(x^{k}\right), w-x^{k}\right\rangle=\varepsilon\left\langle F\left(x^{k}\right), x-x^{k}\right\rangle$, and since $x$ is arbitrary we conclude that $x^{k} \in \operatorname{SOL}(F)$.

From this proposition we conclude that all the sequences $\left\{x^{k}\right\}$ of solutions to $\left(\operatorname{VIP}_{k}\right)$ satisfying $\left\|x^{k}\right\|_{d}=\sigma_{k}$ for all $k$ must be specially studied. We deal with such sequences by using the Basic Lemma. To this end, by following the line of reasoning from [11] we give the next definition.

Definition 3.2. We denote by $\mathcal{W}$ the set of sequences $\left\{x^{k}\right\}$ in $\mathbb{R}_{+}^{n}$, such that for each $k \in \mathbb{N}$,

$$
x^{k} \text { solves problem }\left(\operatorname{VIP}_{k}\right) \text { and }\left\|x^{k}\right\|_{d}=\sigma_{k} .
$$

We point out that the second requirement of (3.1) is verified if $\operatorname{SOL}(F)$ is either empty or unbounded. Indeed, if $\operatorname{SOL}(F)=\emptyset$ then $\left\|x^{k}\right\|_{d}=\sigma_{k}$ for all $k$ by the above reasoning. If $\operatorname{SOL}(F)$ is nonempty and unbounded, then for any $k$ there exists $x^{k} \in \operatorname{SOL}(F)$ such that $\left\|x^{k}\right\|_{d} \geq k$, and then we put $\sigma_{k}=\left\|x^{k}\right\|_{d}$.

If $\mathcal{W}$ is an empty set, then $\operatorname{SOL}(F)$ is nonempty and closed. The latter follows from the continuity of $F$.

The next result generalizes Theorem 3.8.6 from [3], which is a well-known existence theorem for copositive matrices since $q \in \operatorname{int} \operatorname{SOL}(M)^{*}$ iff $\mathrm{W}_{q}(M)=\{0\}$ (see Prop. 2.2), and Theorem 1 from [12] for homogeneous of degree $\gamma>0$ functions.

Theorem 3.3. Let $F$ be c-homogeneous.

(a) If $\mathrm{W}(F)=\{0\}$, then $F \in \mathrm{Q}_{\mathrm{b}}$.

(b) If $F=H+q$ is copositive, and $\mathrm{W}_{q}(H)=\{0\}$, then $\operatorname{SOL}(F)$ is nonempty and compact.

Proof. (a): Let $q \in \mathbb{R}^{n}$ be an arbitrary vector and suppose that there exists $\left\{x^{k}\right\} \in \mathcal{W}$ for the function $F+q$. There is a vector $v$ such that up to subsequences $\frac{x^{k}}{\sigma_{k}} \rightarrow v$, and by (a) of the Basic Lemma for $F^{k}=F+q$ for all $k$, we conclude that $0 \neq v \in \mathrm{W}(F+q)=\mathrm{W}(F)$, a contradiction. Thus, $\operatorname{SOL}(F+q)$ is nonempty and closed. Its boundedness follows from Corollary 2.4(a). Therefore, $F \in \mathrm{Q}_{\mathrm{b}}$ since $q$ was arbitrary.

(b): Suppose that there exists $\left\{x^{k}\right\} \in \mathcal{W}$. There is a vector $v$ such that up to subsequences $\frac{x^{k}}{\sigma_{k}} \rightarrow v$. By (a) of the Basic Lemma for $F^{k}=F$ for all $k$, we conclude that $0 \neq v \in \mathrm{W}_{q}(H)$, a contradiction. Thus, $\operatorname{SOL}(F)$ is nonempty and closed. Its boundedness follows from Corollary 2.4(a).

As a consequence of this theorem we extend and generalize various coercive existence results from the literature: for generalized homogeneous functions (see [37]), for $d$-regular or strictly copositive functions being homogeneous of degree $\gamma>0$ (see [19] and [31] respectively), and for linear $d$-García functions (see [9]). To do this, similarly as in [37], this time for $F$ being $c$-homogeneous, we define the constants $\mu_{\text {inf }}^{*}$ and $\mu_{\text {sup }}^{*}$ as follows:

$$
0 \leq \mu_{\mathrm{inf}}^{*}=\liminf _{\|x\|_{d} \rightarrow+\infty} \frac{|\langle F(x), x\rangle|}{\|x\|_{d} c\left(\|x\|_{d}\right)} \text { and } \mu_{\text {sup }}^{*}=\limsup _{\substack{\|x\|_{d} \rightarrow+\infty \\ x \geq 0}} \frac{|\langle F(x), x\rangle|}{\|x\|_{d} c\left(\|x\|_{d}\right)}<+\infty \text {. }
$$

Corollary 3.4. Let $F$ be c-homogeneous. Then $F \in \mathrm{Q}_{\mathrm{b}}$ under any of the following conditions:

(a) the equation $\langle F(x)-F(0), x\rangle=-\mu$ has no solutions $(x, \mu) \in \Delta_{d} \times\left[\mu_{\mathrm{inf}}^{*}, \mu_{\mathrm{sup}}^{*}\right]$;

(b) $F \in \mathrm{R}(d)=\mathrm{G}(d) \cap \mathrm{R}_{0}$.

Proof. (a): If $0 \neq v \in \mathrm{W}(F)$, then $\langle F(v)-F(0), v\rangle \leq 0$. By hypothesis we may assume that $\|v\|_{d}=1$. Since $\mu_{\mathrm{inf}}^{*} \leq|\langle F(v)-F(0), v\rangle|=-\langle F(v)-F(0), v\rangle \leq \mu_{\mathrm{sup}}^{*}$, we conclude that $(v,\langle F(v)-F(0), v\rangle)$ solves the equation in part (a), a contradiction. Thus, $\mathrm{W}(F)=\{0\}$ and the result follows from Theorem 3.3.

(b): Let $q \in \mathbb{R}^{n}$ be an arbitrary vector and suppose that there exists $\left\{x^{k}\right\} \in \mathcal{W}$ for the function $F+q$. There is a vector $v$ such that up to subsequences $\frac{x^{k}}{\sigma_{k}} \rightarrow v$. By (a) of the Basic Lemma for $F^{k}=F+q$ for all $k$, and since $F+q \in \mathrm{G}(d)$ we get $0 \neq v \in \mathrm{SOL}(F-F(0))$, a contradiction to $F \in \mathrm{R}_{0}$. Therefore, $\operatorname{SOL}(F+q)$ is nonempty and closed. Its boundedness follows from Corollary 2.4(a). Thus, $F \in \mathrm{Q}_{\mathrm{b}}$ since $q$ was arbitrary. 
It is worth pointing out that part (a) holds if $F$ is copositive and $\mu_{\text {inf }}^{*}>0$ whereas part (b) holds if $F$ is strictly copositive (see Ex. 1.3(e)). Moreover, as a consequence of this corollary we generalize Corollary 2.1 from [10] given for the linear complementarity problem.

Corollary 3.5. Let $F$ be a c-homogeneous $\mathrm{G}(d)$-function. It holds that: $F \in \mathrm{Q}_{\mathrm{b}} \Longleftrightarrow F \in \mathrm{R}_{0} \Longleftrightarrow F \in \mathrm{R}(d)$.

Proof. If $F \in \mathrm{Q}_{\mathrm{b}}$, then by Proposition $2.2(\mathrm{~d})$ we get $\operatorname{SOL}(F-F(0))=\{0\}$, thus, $F \in \mathrm{R}_{0}$ and $F \in \mathrm{R}(d)=$ $\mathrm{G}(d) \cap \mathrm{R}_{0}$. If $F \in \mathrm{R}(d)$, then $F \in \mathrm{R}_{0}$ and by the above corollary we conclude that $F \in \mathrm{Q}_{\mathrm{b}}$.

Theorem 3.6. Let $F$ be c-Moré.

(a) If $\mathrm{U}(F)=\{0\}$, then $F \in \mathrm{Q}_{\mathrm{b}}$.

(b) If $F=H+q$ is copositive, and $\mathrm{U}_{q}(H)=\{0\}$, then $\operatorname{SOL}(F)$ is nonempty and compact.

Proof. Proceed similarly as in the proof of Theorem 3.3, this time using (b) of the Basic Lemma and (b) of Corollary 2.4.

As a consequence of this theorem we deduce two coercive existence results from the literature for strongly monotone functions (see [19]) and for strongly copositive functions (see [23]). We also extend a result for strictly copositive functions satisfying the assumption of Ex. 1.3(c) (see [37]).

Corollary 3.7. Consider the following statements:

(a) $F$ is strongly monotone;

(b) $F$ is strongly copositive;

(c) $F$ is c-Moré and strictly copositive;

(d) $F \in \mathrm{Q}_{\mathrm{b}}$.

It holds that $(\mathrm{a}) \Longrightarrow(\mathrm{b}) \Longrightarrow(\mathrm{c}) \Longrightarrow(\mathrm{d})$.

Proof. (a) $\Rightarrow$ (b): It is obvious.

(b) $\Rightarrow(\mathrm{c})$ : Use Proposition 1.5(c).

$(\mathrm{c}) \Rightarrow(\mathrm{d})$ : By hypothesis we have $\mathrm{U}(F)=\{0\}$. The result follows from Theorem 3.6(a).

We next result summarizes some well-known coercive existence results concerning the pseudomonotone case. Condition (a) appears in [24]. Condition (b) is called Crouzeix's condition and appears in [4]. Condition (d) is called Karamardian's condition, appears in [23], and implies the nonemptiness and compactness of $\operatorname{SOL}(F)$ without the pseudomonotonicity assumption. We give a proof by using our approach.

Theorem 3.8. Let $F$ be pseudomonotone. The following four statements are equivalent:

(a) $\operatorname{FEA}_{s}(F) \neq \emptyset$;

(b) $\mathrm{V}(F)=\{0\}$;

(c) $\operatorname{SOL}(F)$ is a nonempty compact convex set;

(d) there exists a compact convex set $K \subseteq \mathbb{R}_{+}^{n}$ such that $\forall x \in \mathbb{R}_{+}^{n} \backslash K \exists z \in K:\langle F(x), z-x\rangle<0$.

Proof. (a) $\Leftrightarrow(\mathrm{b})$ : See Proposition 2.2(b).

$(\mathrm{b}) \Rightarrow(\mathrm{c})$ : Suppose that there exists $\left\{x^{k}\right\} \in \mathcal{W}$. There is a vector $v$ such that up to subsequences $\frac{x^{k}}{\sigma_{k}} \rightarrow v$. By (c) of the Basic Lemma for $F^{k}=F$ for all $k$, we conclude that $0 \neq v \in \mathrm{V}(F)$, a contradiction. Thus, SOL $(F)$ is nonempty and closed. Its boundedness and convexity follow from Corollary 2.4(c) and (2.3) respectively.

$(\mathrm{b}) \Leftarrow(\mathrm{c})$ : It follows from Corollary 2.4(c).

$(\mathrm{c}) \Rightarrow(\mathrm{d})$ : Take $K=\operatorname{SOL}(F)$.

$(\mathrm{d}) \Rightarrow(\mathrm{c})$ : By hypothesis $\mathcal{W}=\emptyset$, thus, $\operatorname{SOL}(F)$ is nonempty and closed. By (d) we also get that $\operatorname{SOL}(F) \subseteq K$.

Corollary 3.9. If $F$ is strictly monotone and $\mathrm{FEA}(F) \neq \emptyset$, then $\mathrm{NCP}(\mathrm{F})$ has a unique solution.

Proof. If $0 \neq v \in \mathrm{V}(F)$, then $v \geq 0$ and $\langle F(x), v\rangle \leq 0$ for all $x \geq 0$. If $\bar{x} \in \mathrm{FEA}(F)$, then $\langle F(\bar{x}), v\rangle \geq 0$ and hence $\langle F(\bar{x}), v\rangle=0$. By hypothesis $\langle F(\bar{x}+v)-F(\bar{x}),(\bar{x}+v)-\bar{x}\rangle>0$, thus, $\langle F(\bar{x}+v), v\rangle>0$, a contradiction. Therefore, $\mathrm{V}(F)=\{0\}$ and $\operatorname{SOL}(F) \neq \emptyset$ by Theorem 3.8. For the uniqueness see Proposition 3.2 from [19]. 
The nonemptiness of the solution set in the above corollary fails to hold if strict monotonicity is weakened to mere monotonicity (see [29]).

\section{Main SEnsitivity AND Stability Results And BOUnds FOR THE SOLUtion SET}

In this section we generalize some results from $[3,9,15]$ where the copositive and pseudomonotone linear cases are studied, and are similar to those from [11] where the multivalued case is studied. The result for $c$-Moré functions have no analog for multifunctions in [11].

Theorem 4.1. If $\bar{F}=\bar{H}+\bar{q}$ is c-homogeneous and $\mathrm{W}_{\bar{q}}(\bar{H})=\{0\}$, then there exists a neighborhood $U$ of $\bar{F}$ and a scalar $r>0$ such that for all $F=H+q$ in $U$ being c-homogeneous, the following assertions hold:

(a) $\mathrm{W}_{q}(H)=\{0\}$;

if in addition, $F$ is copositive then

(b) $\operatorname{SOL}(F)$ is nonempty;

(c) $\|x\|_{d} \leq r$ for all $x \in \mathrm{SOL}(F)$.

Proof. (a): Suppose on the contrary, that there exist $\left\{F^{k}=H^{k}+q^{k}\right\}$ and $\left\{v^{k}\right\}$ such that $\mathbb{D}\left(F^{k}, \bar{F}\right) \rightarrow 0$ and $0 \neq v^{k} \in \mathrm{W}_{q^{k}}\left(H^{k}\right)$. By the $c$-homogeneity assumption we may assume that $\left\|v^{k}\right\|_{d}=1$ (see Eq. (2.1) and Prop. 2.2(d)). Therefore, up to subsequences $v^{k} \rightarrow v$ for some vector $v$. Moreover, since $0 \leq v^{k} \perp$ $H^{k}\left(v^{k}\right)-H^{k}(0) \geq 0$ and $\left\langle H^{k}(0)+q^{k}, v^{k}\right\rangle \leq 0$ for all $k$, by the continuous convergence of $\left\{F^{k}\right\}$ to $\bar{F}$ we conclude that $0 \leq v \perp \bar{H}(v)-\bar{H}(0) \geq 0$ and $\langle\bar{H}(0)+\bar{q}, v\rangle \leq 0$, thus, $0 \neq v \in \mathrm{W}_{\bar{q}}(\bar{H})$, a contradiction.

(b): Use Theorem 3.3 and (a).

(c): On the contrary suppose that there exist $\left\{F^{k}=H^{k}+q^{k}\right\}$ and $\left\{x^{k}\right\}$ such that $\mathbb{D}\left(F^{k}, \bar{F}\right) \rightarrow 0, x^{k} \in \operatorname{SOL}\left(F^{k}\right)$, and $\left\|x^{k}\right\|_{d} \rightarrow+\infty$. Setting $\sigma_{k}=\left\|x^{k}\right\|_{d}$, we deduce that $\frac{x^{k}}{\sigma_{k}} \rightarrow v$ up to subsequences for some vector $v$. Clearly, $x^{k}$ is a solution of $\left(\mathrm{PVIP}_{\mathrm{k}}\right)$ for $F=\bar{F}$ such that $\left\|x^{k}\right\|_{d}=\sigma_{k}$ for all $k$, thus, we can apply (a) of the Basic Lemma for $F=\bar{F}$ and we obtain $0 \neq v \in \mathrm{W}_{\bar{q}}(\bar{H})$, a contradiction.

The next result asserts in particular that the class of $\mathrm{Q}_{\mathrm{b}}$-functions is open in the set of copositive functions from $\mathrm{C}\left(\mathbb{R}_{+}^{n} ; \mathbb{R}^{n}\right)_{c}$. As far as we know, this result does not appear in the literature before.

Corollary 4.2. If $\bar{F} \in \mathrm{Q}_{\mathrm{b}}$, then there exists a neighborhood $U$ of $\bar{F}$ such that $F \in \mathrm{Q}_{\mathrm{b}}$ for all $F \in U$ being copositive.

Proof. Since $\bar{F}$ is $\mathrm{Q}_{\mathrm{b}}$ and $c$-homogeneous, then by Proposition $2.2(\mathrm{~d})$ we conclude that $\operatorname{SOL}(\bar{F}-\bar{F}(0))=\{0\}$. Therefore by $(2.1)$ we get $\mathrm{W}_{-\bar{F}(0)}(\bar{F})=\{0\}$ and from Theorem 4.1, there exists a neighborhood $U$ of $\bar{F}-$ $\bar{F}(0)$ such that $\mathrm{W}_{-F(0)}(F)=\{0\}$ for all $F-F(0) \in U$ being $c$-homogeneous. Thus, $\operatorname{SOL}(F-F(0))=\{0\}$ and by definition $F \in \mathrm{R}_{0}$. If in addition, $F$ is copositive, then $F \in \mathrm{G}(d)$ (see Ex. 1.3(e)) and $F \in \mathrm{Q}_{\mathrm{b}}$ by Corollary 3.4.

Remark 4.3. By proceeding similarly as in Theorem 1 from [13] and using the $c$-homogeneity and the continuous convergence we can prove that the class of regular functions $\mathrm{R}=\cup_{d>0} \mathrm{R}(d)$ is open in $\mathrm{C}\left(\mathbb{R}_{+}^{n} ; \mathbb{R}^{n}\right)_{c}$.

Theorem 4.4. If $\bar{F}=\bar{H}+\bar{q}$ is c-Moré and $\mathrm{U}_{\bar{q}}(\bar{H})=\{0\}$, then there exists a neighborhood $V$ of $\bar{F}$ and a scalar $r>0$ such that for all $F=H+q$ in $V$ being copositive c-Moré, the following assertions hold:

(a) $\mathrm{U}_{q}(H)=\{0\}$;

(b) $\operatorname{SOL}(F)$ is nonempty;

(c) $\|x\|_{d} \leq r$ for all $x \in \operatorname{SOL}(F)$.

Proof. (a): Suppose on the contrary, that there exist $\left\{F^{k}=H^{k}+q^{k}\right\}$ and $\left\{v^{k}\right\}$ such that $\mathbb{D}\left(F^{k}, \bar{F}\right) \rightarrow 0$ and $0 \neq v^{k} \in \mathrm{U}_{q^{k}}\left(H^{k}\right)$. We may assume that $\left\|v^{k}\right\|_{d}=1$. Indeed, since each $H^{k}$ is $c$-Moré copositive (see Rem. 1.2(a)) from $0=\left\langle H^{k}\left(v^{k}\right)-H^{k}(0), v^{k}\right\rangle \geq c\left(\left.\left\|v^{k}\right\|\right|_{d}\right)\left\|v^{k}\right\|_{d}\left\langle H^{k}\left(\frac{v^{k}}{\left\|v^{k}\right\|_{d}}\right)-H^{k}(0), \frac{v^{k}}{\left\|v^{k}\right\|_{d}}\right\rangle \geq 0$ we get $\left\langle H^{k}\left(\frac{v^{k}}{\left\|v^{k}\right\|_{d}}\right)-H^{k}(0), \frac{v^{k}}{\left\|v^{k}\right\|_{d}}\right\rangle=0$. Moreover, $\left\langle H^{k}(0)+q^{k}, \frac{v^{k}}{\left\|v^{k}\right\|_{d}}\right\rangle \leq 0$. Therefore, up to subsequences $v^{k} \rightarrow v$ 
for some vector $v$ and by the continuous convergence of $\left\{F^{k}\right\}$ to $\bar{F}$ we conclude that $0 \neq v \in \mathrm{U}_{\bar{q}}(\bar{H})$, a contradiction.

(b): Use Theorem 3.6 and (a).

(c): We proceed similarly as in the proof of part (c) of Theorem 4.1. If $v$ is the vector of that proof, then by applying (b) of the Basic Lemma for $F=\bar{F}$ we obtain $0 \neq v \in \mathrm{U}_{\bar{q}}(\bar{F})$, a contradiction.

Theorem 4.5. If $\bar{F}$ is pseudomonotone and $\mathrm{V}(\bar{F})=\{0\}$, then there exists a neighborhood $U$ of $\bar{F}$ and a scalar $r>0$ such that for all $F \in U$ the following assertions hold:

(a) $\mathrm{V}(F)=\{0\}$;

if in addition, $F$ is pseudomonotone then

(b) $\operatorname{SOL}(F)$ is nonempty;

(c) $\|x\|_{d} \leq r$ for all $x \in \operatorname{SOL}(F)$.

Proof. (a): Suppose on the contrary, that there exist $\left\{F^{k}\right\},\left\{v^{k}\right\}$ such that $\mathbb{D}\left(F^{k}, \bar{F}\right) \rightarrow 0$ and $0 \neq v^{k} \in \mathrm{V}\left(F^{k}\right)$. We may assume that $\left\|v^{k}\right\|_{d}=1$. Therefore, up to subsequences $v^{k} \rightarrow v$ for some vector $v$. Let us fix $x \geq 0$, by the choice of $v^{k}$ we have $\left\langle F^{k}(x), v^{k}\right\rangle \leq 0$. Taking limit and using the continuous convergence of $\left\{F^{k}\right\}$ to $\bar{F}$ we conclude that $\langle\bar{F}(x), v\rangle \leq 0$, and since $x$ was arbitrary we get $0 \neq v \in \mathrm{V}(\bar{F})$, a contradiction.

(b): Use Theorem 3.8 and (a) above.

(c): We proceed similarly as in the proof of part (c) of Theorem 4.1. If $v$ is the vector of that proof, then by applying (c) of the Basic Lemma for $F=\bar{F}$ we obtain $0 \neq v \in \mathrm{V}(\bar{F})$, a contradiction.

We now establish a continuity property for the solution-set-mapping SOL associated to (NCP).

Theorem 4.6. The mapping $\mathrm{SOL}: \mathrm{C}\left(\mathbb{R}_{+}^{n} ; \mathbb{R}^{n}\right) \rightrightarrows \mathbb{R}^{n}$ is OSC at each $\bar{F}$.

Proof. Let $\left\{\left(F^{k}, x^{k}\right)\right\}$ such that $x^{k} \in \operatorname{SOL}\left(F^{k}\right), \mathbb{D}\left(F^{k}, \bar{F}\right) \rightarrow 0$ and $x^{k} \rightarrow x$. Therefore, $0 \leq x^{k} \perp F^{k}\left(x^{k}\right) \geq 0$. Taking limit and by the continuous convergence of $\left\{F^{k}\right\}$ to $\bar{F}$ we conclude that $x \in \operatorname{SOL}(\overline{\bar{F}})$.

Example 4.7. It is worth pointing out that in general the mapping SOL is not continuous. Indeed, we slightly modify Example 2.2 from [36] and obtain that for

$$
\bar{F}(x)=\left\{\begin{array}{ll}
-4 x+1, & x \in\left[0, \frac{1}{4}[;\right. \\
0, & x \in\left[\frac{1}{4}, \frac{3}{4}[;\right. \\
4 x-3, & x \in\left[\frac{3}{4},+\infty[;\right.
\end{array} \quad \text { and } F^{k}(x)= \begin{cases}-4 x+1, & x \in\left[0, \frac{1}{4}[;\right. \\
\frac{1}{k} x-\frac{1}{4 k}, & x \in\left[\frac{1}{4}, \frac{12 k-1}{16 k-4}[;\right. \\
4 x-3, & x \in\left[\frac{12 k-1}{16 k-4},+\infty[\right.\end{cases}\right.
$$

we have that $\operatorname{SOL}(\bar{F})=\{0\} \cup\left[\frac{1}{4}, \frac{3}{4}\right], \operatorname{SOL}\left(F^{k}\right)=\left\{0 ; \frac{1}{4}\right\}$, and $\left\{F^{k}\right\}$ converges continuously to $\bar{F}$ (see Th. 7.14 from [34]). However, there are no solutions $x^{k}$ in $\operatorname{SOL}\left(F^{k}\right)$ such that $x^{k} \rightarrow \frac{3}{4}$, thus, SOL is not ISC at $\bar{F}$.

We now obtain some estimates for the solution set similar to those from [11] (where the multivalued case is studied) and [28] (where the linear monotone case is studied).

\section{Theorem 4.8.}

(a) If $F$ is c-homogeneous and $M_{F}<0$, then $\operatorname{SOL}(F) \subseteq\left\{x \geq 0: c\left(\|x\|_{d}\right) \leq \max \left(c(0), \frac{\|F(0)\|}{d_{\min } \cdot\left|M_{F}\right|}\right)\right\}$;

(b) if $F$ is c-Moré and $m_{F}>0$, then $\operatorname{SOL}(F) \subseteq\left\{x \geq 0: c\left(\|x\|_{d}\right) \leq \max \left(c(0), \frac{\|F(0)\|}{d_{\min } \cdot m_{F}}\right)\right\}$;

(c) if $F$ is pseudomonotone and $0 \neq \bar{x} \in \mathrm{FEA}_{s}(F)$, then $\operatorname{SOL}(F) \subseteq\left\{x \geq 0:\|x\|_{1} \leq \frac{\langle F(\bar{x}), \bar{x}\rangle}{\min _{1 \leq i \leq n} f_{i}(\bar{x})}\right\}$.

Proof. (a): Assume that $M_{F}<0$ and let $0 \neq x \geq 0$. By Proposition 1.5(b) we get

$$
\langle F(x), x\rangle=\langle F(x)-F(0), x\rangle+\langle F(0), x\rangle \leq M_{F}\|x\|_{d} c\left(\|x\|_{d}\right)+\|F(0)\| \cdot\|x\|
$$

and since $\|x\|_{d} \geq d_{\text {min }} \cdot\|x\|$ we obtain $\langle F(x), x\rangle \leq\|x\|\left\{M_{F} c\left(\|x\|_{d}\right) d_{\text {min }}+\|F(0)\|\right\}$. If $0 \neq x \in \operatorname{SOL}(F)$ then $M_{F} c\left(\|x\|_{d}\right) d_{\min }+\|F(0)\| \geq 0$. 
(b): Assume that $m_{F}>0$ and let $0 \neq x \geq 0$. By Proposition 1.5(a) we get

$$
\langle F(x), x\rangle=\langle F(x)-F(0), x\rangle+\langle F(0), x\rangle \geq m_{F}\|x\|_{d} c\left(\|x\|_{d}\right)-\|F(0)\| \cdot\|x\|
$$

and since $\|x\|_{d} \geq d_{\min } \cdot\|x\|$ we obtain $\langle F(x), x\rangle \geq\|x\|\left\{m_{F} c\left(\|x\|_{d}\right) d_{\min }-\|F(0)\|\right\}$. If $0 \neq x \in \operatorname{SOL}(F)$ then $m_{F} c\left(\|x\|_{d}\right) d_{\min }-\|F(0)\| \leq 0$.

(c): If $x \in \operatorname{SOL}(F)$ then $\langle F(x), \bar{x}-x\rangle \geq 0$. By hypothesis $\langle F(\bar{x}), \bar{x}-x\rangle \geq 0$, which in turn implies $\langle F(\bar{x}), \bar{x}\rangle \geq$ $\langle F(\bar{x}), x\rangle \geq \min _{1 \leq i \leq n} f_{i}(\bar{x})\|x\|_{1}$. The results holds since by hypothesis $\min _{1 \leq i \leq n} f_{i}(\bar{x})>0$.

It is worth pointing out that either the hypothesis of (b) or that of (c) implies that $\mathrm{SOL}(F)$ is nonempty and compact (see Prop. 1.5(e), Cor. 3.7 and Th. 3.8).

\section{PieceWise AFFine CASE}

In this section we study the (NCP) within the class of piecewise affine functions defined on $\mathbb{R}_{+}^{n}$, which we denote by $\mathrm{A}\left(\mathbb{R}_{+}^{n}, \mathbb{R}^{n}\right)$. This class in contained in $\mathrm{C}\left(\mathbb{R}_{+}^{n}, \mathbb{R}^{n}\right)$ (see [34]), thus, our results remain valid.

By proceeding similarly as in [3] we prove that under an homogeneity assumption, the solution-set-mapping behaves as that for the (LCP) (see Cor. 5.5 below).

Firstly, we consider the solution-set-mapping Sol $: \mathbb{R}^{n} \rightrightarrows \mathbb{R}^{n}$ defined by $\operatorname{Sol}(q):=\operatorname{SOL}(\bar{F}+q)$ for $\bar{F}$ being a fixed function from $\mathrm{A}\left(\mathbb{R}_{+}^{n}, \mathbb{R}^{n}\right)$.

\section{Proposition 5.1.}

(a) There exists $\lambda>0$ such that Sol is locally $\operatorname{UL}(\lambda)$ at each $\bar{q} \in \mathbb{R}^{n}$, i.e., there exists a neighborhood $U$ of $\bar{q}$ such that $\operatorname{Sol}(q) \subseteq \operatorname{Sol}(\bar{q})+\lambda\|q-\bar{q}\| \mathbb{B}$ for all $q \in U$;

(b) if $\operatorname{Sol}(\bar{q})$ is bounded, then $\operatorname{Sol}$ is usc at $\bar{q}$. Moreover, there exists a constant $r>0$ and a neighborhood $U$ of $\bar{q}$ such that for all $q \in U$ it holds that $\|x\| \leq r$ for all $x \in \operatorname{Sol}(q)$.

Proof. (a): It is sufficient to prove that Sol is piecewise polyhedral since by Robinson's result (see [33]) a piecewise polyhedral multifunction is locally $\mathrm{UL}(\lambda)$ for some $\lambda>0$. Indeed, gph $\bar{F}=\bigcup_{i=1}^{m} P_{i}$ where each $P_{i} \subseteq \mathbb{R}^{n} \times \mathbb{R}^{n}$ is a polyhedral set. We define the set $\Sigma=\{(q, x, y): x \geq 0, y=\bar{F}(x), y+q \geq 0,\langle y+q, x\rangle=0\}$. If $\bar{J}=I \backslash J$, then $\Sigma=\bigcup_{i=1}^{m} \bigcup_{J \subseteq I} X_{i, J}$ where $X_{i, J}:=\left\{(q, x, y):(x, y) \in P_{i},(y+q)_{J}=0,(y+q)_{\bar{J}} \geq 0, x_{\bar{J}}=0\right\}$ is a polyhedral set and hence $\Sigma$ is piecewise polyhedral. If $\Pi$ is the orthogonal projection defined by $\Pi(q, x, y):=(q, x)$ then gph Sol $=\Pi(\Sigma)$. Therefore, gph Sol is a finite union of polyhedral sets.

(b): We proceed similarly as in Corollary 7.2.3 from [3]. If $\operatorname{Sol}(\bar{q})$ is empty, so is $\operatorname{Sol}(q)$ for $q$ sufficiently close to $\bar{q}$ and the result holds vacuously. If $\operatorname{Sol}(\bar{q})$ is nonempty, then since it is bounded and closed there exists an open set $V=r \operatorname{int} \mathbb{B}$ with $r>0$ such that by restricting $U$ from (a) if necessary, we get $\operatorname{Sol}(q) \subseteq \operatorname{Sol}(\bar{q})+\lambda\|q-\bar{q}\| \mathbb{B} \subseteq V$ for all $q \in U$. Thus, Sol is usc at $\bar{q}$ and $\operatorname{Sol}(q) \subseteq r \mathbb{B}$ for all $q \in U$.

Remark 5.2. (a) Part (a) of the above proposition implies that $\operatorname{NCP}(\bar{F})$ is semistable, i.e., for every open set $U$ containing $\operatorname{SOL}(\bar{F})$, there exist scalars $\mu, \varepsilon>0$ such that for every continuous function $F$ satisfying $\omega=\sup _{x \in \mathbb{R}_{+}^{n} \cap \operatorname{cl} U}\|F(x)-\bar{F}(x)\|<\varepsilon$, it holds that $\operatorname{SOL}(F) \cap U \subseteq \operatorname{SOL}(\bar{F})+\mu \omega \mathbb{B}$. In fact, NCP $(\bar{F})$ is semistable iff Sol is locally $\operatorname{UL}(\lambda)$ at 0 (see Prop. 5.5.5 from [8]).

(b) If $\bar{F}$ is linear and $\operatorname{Sol}(q) \neq \emptyset$ for all $q \in \mathbb{R}^{n}$, then Sol is Lipschitzian iff Sol is single-valued (see [17]).

(c) Part (a) of the above proposition implies that Sol is calm at every $\bar{q} \in \operatorname{dom}$ Sol (see [34]). Moreover, since $\bar{F} \in \mathrm{A}\left(\mathbb{R}_{+}^{n}, \mathbb{R}^{n}\right)$ the set dom Sol is closed (see Prop. 3 from [14]).

Secondly, we study the solution-set-mapping SOL : $\mathrm{A}\left(\mathbb{R}_{+}^{n}, \mathbb{R}^{n}\right)_{c} \rightrightarrows \mathbb{R}^{n}$ defined on the set of $c$-homogeneous piecewise affine functions on $\mathbb{R}_{+}^{n}$. To this end, we shall employ the equivalent metric $\mathbb{D}_{\mathrm{o}}$. 
Theorem 5.3. Let $c$ be nondecreasing. If $\bar{F}=\bar{H}+\bar{q} \in \mathrm{A}\left(\mathbb{R}_{+}^{n} ; \mathbb{R}^{n}\right)_{c}$ and $\mathrm{W}_{\bar{q}}(\bar{H})=\{0\}$, then there exist scalars $r, \mu>0$ and a neighborhood $V$ of $\bar{F}$ in $\left(\mathrm{A}\left(\mathbb{R}_{+}^{n} ; \mathbb{R}^{n}\right)_{c}, \mathbb{D}_{\mathrm{o}}\right)$ such that for all $F=H+q \in V$ being copositive, the following assertions hold:

(a) $\operatorname{SOL}(F)$ is nonempty;

(b) $\|x\|_{d} \leq r$ for all $x \in \mathrm{SOL}(F)$;

(c) $\operatorname{SOL}(\bar{F}) \subseteq \operatorname{SOL}(\bar{F})+\mu \mathbb{D}_{\mathrm{o}}(F, \bar{F}) \mathbb{B}$.

Proof. (a)-(b): By Theorem 4.1 there exists a neighborhood $U$ of $\bar{F}$ such that parts (a) and (b) hold in $U$. By (b) of Proposition 1.8 the same holds in a neighborhood $V$ of $\bar{F}$ on $\left(\mathrm{A}\left(\mathbb{R}_{+}^{n} ; \mathbb{R}^{n}\right)_{c}, \mathbb{D}_{\mathrm{o}}\right)$.

(c): We proceed similarly as in Theorem 7.5.1 from [3]. Let $F \in V$ be copositive and $x \in \operatorname{SOL}(F)$. Therefore, $0 \leq x \perp F(x) \geq 0$ and $\|x\|_{d} \leq r$ by (b). For $p=F(x)-\bar{F}(x)$ we have $0 \leq x \perp \bar{F}(x)+p \geq 0$, thus, $x \in \operatorname{Sol}(p)=$ $\operatorname{SOL}(\bar{F}+p)$. By Proposition 1.8(a) and since $c$ is nondecreasing we have $\|p\|_{\infty} \leq c(r) \mathbb{D}_{\mathrm{o}}(F, \bar{F})+\|F(0)-\bar{F}(0)\| \|_{\infty}$, thus, $p$ can be made arbitrarily close to 0 by restricting $V$ if necessary and by Proposition 5.1 we conclude that there exists $\lambda>0$ such that $\operatorname{Sol}(p) \subseteq \operatorname{Sol}(0)+\lambda\|p\| \mathbb{B}$. Since $\operatorname{Sol}(0)=\operatorname{SOL}(\bar{F})$ and $\|p\| \leq \sqrt{n}\|p\|_{\infty}$ we conclude that $\operatorname{Sol}(p) \subseteq \operatorname{SOL}(\bar{F})+\lambda \sqrt{n}\left[c(r) \mathbb{D}_{\mathrm{o}}(F, \bar{F})+\|F(0)-\bar{F}(0)\|_{\infty}\right] \mathbb{B}$, thus, $x$ is in the right-hand side set. Since $x$ was arbitrary, then part (c) holds for $\mu=\lambda \sqrt{n} \max \{1, c(r)\}$.

Remark 5.4. Under the hypotheses of the above theorem and as a consequence of it, we conclude that the following properties hold within the class of copositive mappings from $\mathrm{A}\left(\mathbb{R}_{+}^{n} ; \mathbb{R}^{n}\right)_{c}$ :

(a) There exists $\mu>0$ such that for every $x \in \operatorname{SOL}(F)$ for $F$ close to $\bar{F}$, it holds that $\mathrm{d}(x, \operatorname{SOL}(\bar{F})) \leq \mu \mathbb{D}_{\mathrm{o}}(F, \bar{F})$. This is the so-called local error bound property and is known to be equivalent to part (c) of the theorem (see [17]). (b) For every $\varepsilon>0$ there exists $\delta>0$ such that $\operatorname{SOL}(F) \cap(\operatorname{SOL}(\bar{F})+\varepsilon \mathbb{B}) \neq \emptyset$ for every $F$ satisfying $\mathbb{D}_{\mathrm{o}}(F, \bar{F})<\delta$. This property is called stability of $\operatorname{NCP}(\bar{F})$ and generalizes Corollary 6 from [16] valid for the affine case.

For $(\mathrm{LCP})$ it is common to denote by $\operatorname{SOL}(q, M)$ and $\operatorname{FEA}_{s}(q, M)$ its solution and strictly feasibility sets respectively. As a consequence of the preceding theorem we deduce Theorem 7.5.1 from [3].

Corollary 5.5. If $\bar{M} \in \mathbb{R}^{n \times n}$ and $\bar{q} \in \operatorname{int} \operatorname{SOL}(0, \bar{M})^{*}$, then there exist scalars $\varepsilon, r, \mu>0$, such that for all $q$ and $M$ being copositive satisfying $\|M-\bar{M}\|+\|q-\bar{q}\|<\varepsilon$, the following statements hold:

(a) $\operatorname{SOL}(q, M)$ is nonempty;

(b) $\|x\| \leq r$ for all $x \in \operatorname{SOL}(q, M)$;

(c) $\operatorname{SOL}(q, M) \subseteq \operatorname{SOL}(\bar{q}, \bar{M})+\mu(\|M-\bar{M}\|+\|q-\bar{q}\|) \mathbb{B}$.

Proof. By Example 1.3 and Proposition 2.2(a) $F(x)=M x+q$ and $\bar{F}(x)=\bar{M} x+\bar{q}$ satisfy the hypotheses of Theorem 5.3 for any $d>0$ and $c(\lambda)=\lambda$. Moreover, the metric $\mathbb{D}_{\circ}(F, \bar{F})=\|M-\bar{M}\|_{d, \infty}+\|q-\bar{q}\|_{\infty}$ where $\|\cdot\|_{d, \infty}$ is the matrix norm induced by $\|\cdot\|_{d}$ and $\|\cdot\|_{\infty}$ is equivalent to $\|M-\bar{M}\|+\|q-\bar{q}\|$.

The above corollary is related to several characterizations of bounded solutions for copositive-plus matrices (see [6], Th. 2 of [27] and Cor. 7.5.2 of [3]). Let $\bar{M}$ be copositive-plus i.e., $\langle\bar{M} x, x\rangle \geq 0$ for all $x \geq 0$ and $\left[x \geq 0,\langle\bar{M} x, x\rangle=0 \Rightarrow\left(\bar{M}+\bar{M}^{\top}\right) x=0\right]$. The following equivalences hold: $\operatorname{SOL}(\bar{q}, \bar{M}) \neq \emptyset$ and bounded iff $\bar{q} \in \operatorname{int} \operatorname{SOL}(0, \bar{M})^{*}$ iff $\operatorname{FEA}_{s}(\bar{q}, \bar{M}) \neq \emptyset$ (see Cor. 6.3 of [9]) iff there exists a scalar $\varepsilon>0$ such that $\operatorname{SOL}(q, M) \neq \emptyset$ for all $q$ and $M$ satisfying $\|M-\bar{M}\|+\|q-\bar{q}\|<\varepsilon$ iff there exist scalars $\varepsilon, r>0$ such that $\operatorname{SOL}(q, M) \neq \emptyset$ for all $q$ and $M$ copositive satisfying $\|M-\bar{M}\|+\|q-\bar{q}\|<\varepsilon$, and furthermore $\|x\| \leq r$ for all $x \in \operatorname{SOL}(q, M)$.

Acknowledgements. The author is greatly indebted to the referees for several helpful and constructive comments concerning an earlier version of this paper. 


\section{REFERENCES}

[1] J.-P. Aubin and H. Frankowska, Set-Valued Analysis. Birkhäuser, Boston (1990).

[2] A. Auslender and M. Teboulle, Asymptotic Cones and Functions in Optimization and Variational Inequalities. Springer, Berlin (2003).

[3] R.W. Cottle, J.S. Pang and R.E. Stone, The Linear Complementarity Problem. Academic Press, New York (1992).

[4] J.P. Crouzeix, Pseudomonotone variational inequality problems: Existence of solutions. Math. Program. 78 (1997) 305-314.

[5] S. Dafermos, Sensitivity analysis in variational inequalities. Math. Oper. Res. 13 (1988) 421-434.

[6] R. Doverspike, Some perturbation results for the linear complementarity problem. Math. Program. 23 (1982) 181-192.

[7] F. Facchinei and J.S. Pang, Total stability of variational inequalities. Technical Report 09-98, Dipartimento di Informatica e Sistematica, Università Degli Stuti di Roma "La Sapienza" (1998).

[8] F. Facchinei and J.S. Pang, Finite-Dimensional Variational Inequalities and Complementarity Problems I. Springer, New York (2003).

[9] F. Flores-Bazán and R. López, The linear complementarity problem under asymptotic analysis. Math. Oper. Res. 30 (2005) 73-90.

[10] F. Flores-Bazán and R. López, Characterizing Q-matrices beyong L-matrices. J. Optim. Theory Appl. 127 (2005) $447-457$.

[11] F. Flores-Bazán and R. López, Asymptotic analysis, existence and sensitivity results for a class of multivalued complementarity problems. ESAIM: COCV 12 (2006) 271-293.

[12] M.S. Gowda, Complementarity problems over locally compact cones. SIAM J. Control Optim. 27 (1989) 836-841.

[13] M.S. Gowda and J.S. Pang, On solution stability of the linear complementarity problems. Math. Oper. Res. 17 (1992) $77-83$.

[14] M.S. Gowda and J.S. Pang, Some existence results for multivalued complementarity problems. Math. Oper. Res. 17 (1992) $657-669$.

[15] M.S. Gowda and J.S. Pang, The basic theorem of complementarity revisited. Math. Program. 58 (1993) 161-177.

[16] M.S. Gowda and J.S. Pang, On the boundedness and stability to the affine variational inequality problem. SIAM J. Control Optim. 32 (1994) 421-441.

[17] M.S. Gowda and R. Sznajder, On the Lipschitzian properties of polyhedral multifunctions. Math. Program. 74 (1996) $267-278$.

[18] C.D. Ha, Application of degree theory in stability of the complementarity problem. Math. Oper. Res. 12 (1987) 368-376.

[19] P.T. Harker and J.S. Pang, Finite-dimensional variational and nonlinear complementarity problems: A survey of theory, algorithms and applications. Math. Program. 48 (1990) 161-220.

[20] W.W. Hogan, Point-to-set maps in mathematical programming. SIAM Rev. 15 (1973) 591-603.

[21] G. Isac, The numerical range theory and boundedness of solutions of the complementarity problem. J. Math. Anal. Appl. 143 (1989) 235-251.

[22] G. Isac, Pseudo-monotone complementarity problems in Hilbert space. J. Optim. Theory Appl. 75 (1992) 281-295.

[23] S. Karamardian, Generalized complementarity problem. J. Optim. Theory Appl. 8 (1971) 161-168.

[24] S. Karamardian, Complementarity problems over cones with monotone and pseudomonotone maps. J. Optim. Theory Appl. 18 (1976) 445-454.

[25] D. Kinderlehrer and G. Stampacchia, An Introduction to Variational Inequalities and Their Applications. Academic Press, New York (1980).

[26] J. Kyparisis, Sensitivity analysis for variational inequalities and complementarity problems. Ann. Oper. Res. 27 (1990) $143-174$.

[27] O.L. Mangasarian, Characterizations of bounded solutions of linear complementarity problems. Math. Program. Study 19 (1982) 153-166.

[28] O.L. Mangasarian and L. McLinden, Simple bounds for solutions of monotone complementarity problems and convex programs. Math. Program. 32 (1985) 32-40.

[29] N. Megiddo, A monotone complementarity problem with feasible solutions but no complementarity solutions. Math. Program. 12 (1977) 131-132.

[30] N. Megiddo, On the parametric nonlinear complementarity problem. Math. Program. Study 7 (1978) 142-150.

[31] J.J. Moré, Coercivity conditions in nonlinear complementarity problems. SIAM Rev. 17 (1974) 1-16.

[32] J.S. Pang, Complementarity problems, in Nonconvex Optimization and its Applications: Handbook of Global Optimization, R. Horst and P.M. Pardalos Eds., Kluwer, Dordrecht (1995).

[33] S.M. Robinson, Some continuity properties of polyhedral multifunctions. Math. Program. Study 14 (1981) 206-214.

[34] R.T. Rockafellar and R.J. Wets, Variational Analysis. Springer, Berlin (1998).

[35] R.L. Tobin, Sensitivity analysis for complementarity problems. J. Optim. Theory Appl. 48 (1986) 191-204.

[36] S.W. Xiang and Y.H. Zhou, Continuity properties of solutions of vector optimization. Nonlinear Anal. 64 (2006) $2496-2506$.

[37] Y. Zhao, Existence of a solution to nonlinear variational inequality under generalized positive homogeneity. Oper. Res. Lett. 25 (1999) 231-239. 